\title{
Clinical Practice of Adjuvant Chemotherapy in Patients with Early-Stage Epithelial Ovarian Cancer
}

\author{
Lindy M.J. Frielink ${ }^{a, b}$ Brenda M. Pijlman ${ }^{b, c}$ Nicole P.M. Ezendam ${ }^{c, d}$ \\ Johanna M.A. Pijnenborg ${ }^{c, e}$ \\ aDepartment of General Practice, Faculty of Health, Medicine and Life Sciences, Maastricht University, Maastricht, \\ ${ }^{b}$ Department of Obstetrics and Gynecology, Jeroen Bosch Hospital, 's-Hertogenbosch, ${ }^{c}$ Comprehensive Cancer \\ Centre The Netherlands, Eindhoven, ${ }^{\mathrm{d}}$ Department of Medical and Clinical Psychology, CoRPS - Center of Research \\ on Psychology in Somatic Diseases, Tilburg University, and e Department of Obstetrics and Gynecology, \\ Elisabeth-TweeSteden Hospital, Tilburg, The Netherlands
}

\section{Key Words}

Adjuvant chemotherapy Clinical practice $\cdot$ Early-stage

cancer · Epithelial ovarian cancer

\section{Abstract}

Background: Adjuvant platinum-based chemotherapy improves survival in women with early-stage epithelial ovarian cancer (EOC). Yet, there is a wide variety in clinical practice. Methods: All patients diagnosed with FIGO I and Ila EOC (2006-2010) in the south of the Netherlands were analyzed. The percentage of patients that received adjuvant chemotherapy was determined as well as the comprehensiveness of staging and outcome. Results: Forty percent (54/135) of the patients with early-stage EOC received adjuvant chemotherapy. Treatment with adjuvant chemotherapy was associated with FIGO stage, clear-cell histology and nonoptimal staging. Optimal staging was achieved in 50\%, and nonoptimal staging was associated with advanced age, comorbidity and treatment in a non-referral hospital. Overall, there was no difference in outcome between patients with and without adjuvant chemotherapy. Yet, in grade 3 tumors, adju-

\section{KARGER}

E-Mail karger@karger.com www.karger.com/che

\section{(c) 2016 The Author(s) \\ Published by S. Karger AG, Basel 0009-3157/16/0616-0287\$39.50/0}

This article is licensed under the Creative Commons AttributionNonCommercial-NoDerivatives 4.0 International License (CC BY NC-ND) (http://www.karger.com/Services/OpenAccessLicense) Usage and distribution for commercial purposes as well as any distribution of modified material requires written permission. vant chemotherapy seems beneficial. Conclusions: Selective treatment of patients with early-stage EOC might reduce adjuvant chemotherapy without compromising outcome.

(C) 2016 The Author(s)

Published by S. Karger AG, Basel

\section{Introduction}

Each year, approximately 1,200 women are diagnosed with epithelial ovarian cancer (EOC) in the Netherlands, and 1,000 patients die of the disease [1,2]. The high mortality rate is explained by the fact that the majority of cases are diagnosed at an advanced stage of the disease: FIGO IIb-IV [3]. Approximately one third of the patients are diagnosed at an early stage: FIGO I-IIa [4]. The prognosis of EOC is mainly related to FIGO stage, with 5-year relative survival rates ranging from 68 to $90 \%$ in early-stage patients compared to $13-60 \%$ in advanced-stage disease [5]. Patients with early-stage disease should undergo an optimal staging procedure, including iliac and para-aortic lymph node sampling, omentectomy, peritoneal washing and biopsies of the peritoneum. In patients with non- 
optimal staging, adjuvant chemotherapy is generally suggested to reduce the risk of recurrence from occult residual disease. In optimally staged patients, adjuvant chemotherapy is withheld in the majority of cases. Two clinical trials, the ICON1 and ACTION trials, established the current clinical guidelines for the treatment of ovarian cancer $[6,7]$. These trials, which were published in 2003 and included over 400 patients, demonstrated that the survival of patients with early-stage EOC improves when adjuvant chemotherapy is added to the surgical procedure. However, the ACTION trial, which analyzed subgroups of patients with optimal and nonoptimal staging, showed that the benefits of adjuvant chemotherapy are limited to nonoptimally staged patients. Based on the recently updated systematic review by Lawrie et al. [8], there is high-quality evidence that adjuvant platinumbased chemotherapy is effective in prolonging survival in women with early-stage EOC. Yet, it remains uncertain whether women with low- and intermediate-risk earlystage disease will benefit as much from adjuvant chemotherapy as women with high-risk disease. Recent data demonstrated that even 12 years after the end of chemotherapy neuropathy symptoms were experienced by $51 \%$ of the patients with ovarian cancer [9]. Hence, as mentioned by the authors in the Cochrane review, adjuvant chemotherapy in women with lower-risk EOC should be individualized. This indicates that there might be a wide variety in clinical practice regarding the application of adjuvant chemotherapy in early-stage EOC.

The aim of the present observational study was to determine the use of adjuvant chemotherapy in clinical practice for the treatment of patients with early-stage EOC with respect to the staging procedure and highgrade tumors.

\section{Patients and Methods}

\section{Setting}

In the Netherlands, gynecologic cancer care is organized by regional cooperating hospitals and controlled by tertiary centers specialized in oncology. The Gynecological Oncology Center South (GOCS) is a collaborating organization for the registration and management of oncological patients in the southern part of the Netherlands. The GOCS comprises ten collaborating hospitals, including two referral hospitals. The collaborating hospitals use a web-based data registration system to register all patients with gynecologic malignancies within this region.

\section{Patient Selection}

Patients with FIGO stage I-IIa EOC diagnosed between January 2006 and December 2010 at GOCS were retrospectively analyzed. The local research committee approved the study. The on- line files of all patients treated for early-stage EOC within GOCS were reviewed for inclusion. A total of 189 patients were selected. Data were collected from the patients' medical charts in each hospital. Patients with non-EOC, borderline pathology, ovarian metastasis from other malignancies and advanced-stage disease were excluded, resulting in 135 patients included for evaluation.

\section{Patient Characteristics}

Patient characteristics (i.e. age, body mass index, comorbidities and utilization of anticoagulants), the surgical procedure performed and histology results were recorded. Tumor grade was defined as well differentiated (grade 1), moderately differentiated (grade 2) and poorly differentiated (grade 3 ). The comprehensiveness of the staging procedure was evaluated. Optimal staging was defined as bilateral salpingo-oophorectomy, hysterectomy, omentectomy, comprehensive lymph node sampling of at least ten lymph nodes, peritoneal sampling and sampling of peritoneal fluid. In patients of reproductive age who wished to preserve fertility, bilateral salpingo-oophorectomy and hysterectomy were omitted. In some patients $(\mathrm{n}=16)$ who were optimally staged, including all the steps of the staging procedure, sampling of $\geq 10$ lymph nodes could not be achieved. Since these patients underwent an adequate staging procedure, they were considered as optimally staged patients if 5-10 lymph nodes were sampled. Data collected included the participation of a gynecologic oncologist at the time of surgery, use of adjuvant chemotherapy and follow-up data including recurrent disease and survival.

\section{Outcomes}

Primary outcome was the percentage of patients who received adjuvant chemotherapy after surgery. According to Dutch guidelines, all nonoptimally staged patients are indicated for adjuvant chemotherapy. In optimally staged patients, the indications for adjuvant chemotherapy were examined. Secondary outcomes were as follows: (1) the percentage of patients who underwent a comprehensive staging procedure; (2) reasons for incomplete surgery if surgical staging was omitted; (3) recurrence-free survival (RFS), and (4) overall survival (OS).

\section{Statistical Analysis}

Data were analyzed using SPSS Statistics 20.0 software (IBM, Armonk, N.Y., USA). Univariate analysis of categorical data was performed using the $\chi^{2}$ test. ANOVA was used for univariate anal$y$ sis of continuous variables. Two-sided $p$ values $<0.05$ were considered statistically significant. To identify predictors of the use of adjuvant chemotherapy and the comprehensiveness of the staging procedure, multivariate logistic regression analyses were performed. Outcomes were described by odds ratio (OR) and 95\% confidence interval (CI). Survival curves for RFS and OS were estimated by the Kaplan-Meier method and compared by the logrank test. Based on literature evidence that the effect of adjuvant chemotherapy depends on the comprehensiveness of the staging procedure, subgroup analysis was performed by considering optimally and nonoptimally staged patients separately. Subgroup analysis was also performed for patients with grade 3 tumors, since our aim was to investigate adjuvant chemotherapy in grade 3 tumors as a result of the lack of consensus in current guidelines. The numbers were too small for logistic regression. The Cox proportional hazard model was used to calculate the hazard ratio (HR) with correction for the staging procedure. 
Fig. 1. Flowchart of treatment.

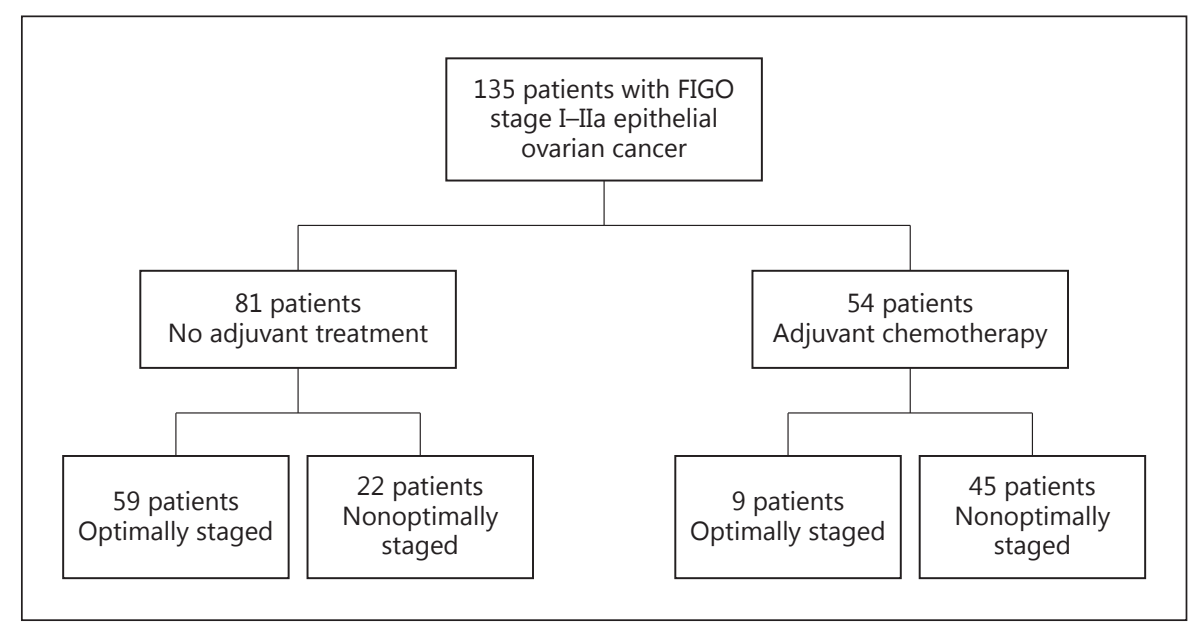

\section{Results}

\section{Patients}

The primary and adjuvant treatments received by all patients $(n=135)$ are listed in figure 1 . All patients underwent a surgical procedure, and the majority (84\%) were treated in accordance with Dutch guidelines. Fiftyfour patients (40\%) received adjuvant chemotherapy. Patient characteristics are shown in table 1. Patients treated with adjuvant chemotherapy differed significantly from those who did not receive adjuvant treatment with respect to FIGO stage $(\mathrm{p}<0.001)$, tumor grade $(\mathrm{p}=0.002)$, histology $(\mathrm{p}<0.001)$, staging performance $(\mathrm{p}<0.001)$ and treatment in a referral hospital $(\mathrm{p}=0.019)$.

\section{Adjuvant Chemotherapy}

All 54 patients who underwent adjuvant treatment received platinum-based chemotherapy. The majority of these patients $(n=49)$ received a combination of carboplatin and paclitaxel, and 4 patients received carboplatin as single-agent chemotherapy. One patient received cyclophosphamide, Adriamycin and cisplatin, since a lymphoma was simultaneously diagnosed.

In multivariate logistic regression analysis (table 2), the application of adjuvant chemotherapy was associated with FIGO stage Ic and IIa (OR: 13.2; 95\% CI, 4.2-41.4), clear-cell histology (OR: 19.1; 95\% CI, 2.0-184) and nonoptimal staging (OR: 11.1; 95\% CI, 3.6-34.2). Patients who received adjuvant chemotherapy after optimal staging $(\mathrm{n}=9)$ were diagnosed with either FIGO Ic/IIa $(\mathrm{n}=$ $4)$, grade $3(n=1)$ or both $(n=3)$. Only 1 patient with FIGO Ia and grade 1, received adjuvant chemotherapy after a complete staging.

Chemotherapy in Early-Stage Ovarian Cancer

\section{Staging Procedure}

Overall, $50 \%(n=68)$ of the patients were optimally staged, of whom $44 \%(n=36)$ underwent a restaging procedure. Advanced age $(p<0.001)$, high comorbidity (classification 3 and 4 of the American Society of Anesthesiologists; $\mathrm{p}=0.001)$ and anticoagulant treatment $(\mathrm{p}=$ 0.003 ) were significantly associated with a nonoptimal staging procedure. Participation of a gynecologic oncologist at the time of surgery $(\mathrm{p}=0.008)$ and treatment in a referral hospital $(\mathrm{p}=0.006)$ were associated with a higher rate of optimal staging. Knowledge of tumor grade was not associated with less comprehensive staging or restaging procedures (table 3 ).

In multivariate logistic regression analysis, age (OR: 1.1; 95\% CI, 1.0-1.1), high comorbidity (OR: 12.7; 95\% CI, 1.5-106) and surgery performed in non-referral hospitals (OR: 6.3; 95\% CI, 2.0-20.2) were associated with less optimal staging.

As shown in figure 1 , a total of 22 nonoptimally staged patients did not receive adjuvant treatment. The majority of these patients were diagnosed with grade 1 tumors $(\mathrm{n}=12)$, and a minority with grade $2(\mathrm{n}=4)$ or grade 3 tumors $(n=6)$. In 13 cases, the reasons for omitting adjuvant chemotherapy in nonoptimally staged patients were included in the patient charts. In addition to tumor characteristics, these reasons were advanced age $(n=2)$, comorbidity $(n=3)$ and refusal of the patient $(n=6)$, although in some cases, multiple reasons were applicable. In 2 cases, adjuvant chemotherapy was omitted based on perioperative assessment. 
Table 1. Baseline characteristics

\begin{tabular}{|c|c|c|c|c|}
\hline \multirow[t]{2}{*}{ Characteristics } & \multicolumn{2}{|c|}{ Adjuvant chemotherapy, $\mathrm{n}(\%)$} & \multirow[t]{2}{*}{ Total } & \multirow[t]{2}{*}{$\mathrm{p}$ value } \\
\hline & no & yes & & \\
\hline Patients & $81(60)$ & $54(40)$ & 135 & \\
\hline Mean age $(S D)$, years $(n=135)$ & $59.1(15.8)$ & $58.7(11.5)$ & & 0.861 \\
\hline \multicolumn{5}{|l|}{ Body mass index $(\mathrm{n}=95)$} \\
\hline$<25$ & $21(41)$ & $20(45)$ & 41 & \\
\hline $25-30$ & $12(24)$ & $17(39)$ & 29 & \\
\hline$\geq 30$ & $18(35)$ & $7(16)$ & 25 & 0.068 \\
\hline \multicolumn{5}{|l|}{ Comorbidity $(\mathrm{n}=132)$} \\
\hline ASA $1-2$ & $69(88)$ & $48(89)$ & 117 & \\
\hline ASA 3-4 & $9(12)$ & $6(11)$ & 15 & 1.00 \\
\hline \multicolumn{5}{|l|}{ FIGO stage $(\mathrm{n}=135)$} \\
\hline Ia & $57(70)$ & $8(15)$ & 65 & \\
\hline $\mathrm{Ib}$ & $2(3)$ & $3(6)$ & 5 & \\
\hline Ic & $22(27)$ & $40(74)$ & 62 & \\
\hline IIa & $0(0)$ & $3(6)$ & 3 & $<0.001$ \\
\hline \multicolumn{5}{|l|}{ Tumor grade $^{\mathrm{a}}(\mathrm{n}=135)$} \\
\hline Grade 1 & $34(42)$ & $9(17)$ & 43 & \\
\hline Grade 2 & $27(33)$ & $22(41)$ & 49 & \\
\hline Grade 3 & $15(19)$ & $21(39)$ & 36 & \\
\hline Missing & $5(6)$ & $2(4)$ & 7 & 0.002 \\
\hline \multicolumn{5}{|l|}{ Histology $(\mathrm{n}=135)$} \\
\hline Serous & $38(47)$ & $22(41)$ & 60 & \\
\hline Mucinous & $22(27)$ & $2(4)$ & 24 & \\
\hline Endometrioid & $16(20)$ & $14(26)$ & 30 & \\
\hline Clear cell & $4(5)$ & $14(26)$ & 18 & \\
\hline Malignant Brenner & $0(0)$ & $1(2)$ & 3 & \\
\hline Other & $1(1)$ & $1(2)$ & 2 & $<0.001$ \\
\hline \multicolumn{5}{|l|}{ Staging performance $(\mathrm{n}=135)$} \\
\hline Optimal & $59(73)$ & $9(17)$ & 68 & \\
\hline Nonoptimal & $22(27)$ & $45(83)$ & 67 & $<0.001$ \\
\hline \multicolumn{5}{|l|}{ Surgery $(n=135)$} \\
\hline 1 operation & $51(63)$ & $48(89)$ & 99 & \\
\hline$>1$ operation & $30(37)$ & $6(11)$ & 36 & 0.002 \\
\hline \multicolumn{5}{|l|}{ Type of hospital $(\mathrm{n}=135)$} \\
\hline Referral hospital & $20(25)$ & $4(7)$ & 24 & \\
\hline Non-referral hospital & $61(75)$ & $50(93)$ & 111 & 0.019 \\
\hline \multicolumn{5}{|c|}{ Gynecologic oncologist at surgery $(n=135)$} \\
\hline Attendant & $54(74)$ & $30(63)$ & 84 & \\
\hline Absent & $19(26)$ & $18(37)$ & 37 & 0.237 \\
\hline \multicolumn{5}{|l|}{ Recurrence $(\mathrm{n}=131)$} \\
\hline No recurrence & $63(81)$ & $43(81)$ & 106 & \\
\hline Recurrence & $15(19)$ & $10(19)$ & 25 & 1.00 \\
\hline \multicolumn{5}{|l|}{ Survival $(\mathrm{n}=128)$} \\
\hline Patients alive & $64(83)$ & $42(82)$ & 106 & \\
\hline Deaths & $13(17)$ & $9(18)$ & 22 & 1.00 \\
\hline
\end{tabular}




\section{Recurrence and Survival}

The median follow-up was 40.5 months. Recurrent disease was detected in $19 \%$ of the patients $(n=25)$ and uniformly distributed between the adjuvant chemotherapy and no adjuvant chemotherapy groups (table 1). Recurrence occurred in 9 grade 1 tumors, 7 grade 2 tumors

Table 2. Logistic regression: correlates for adjuvant chemotherapy

\begin{tabular}{lrl}
\hline Variable & OR & $95 \%$ CI \\
\hline FIGO stage Ic-IIa (vs. Ia and Ib) & 13.2 & $4.2-41.4$ \\
Grade 3 (vs. grade 1 and 2) & 1.2 & $0.3-4.7$ \\
Clear-cell tumors (vs. non-clear-cell tumors) & 19.1 & $2.0-184$ \\
Nonoptimal staging (vs. optimal staging) & 11.1 & $3.6-34.2$ \\
Non-referral hospitals (vs. referral hospitals) & 1.2 & $0.3-5.1$ \\
\hline
\end{tabular}

Nagelkerke $\mathrm{R}^{2}: 0.61$. and 8 grade 3 tumors. The Kaplan-Meier curves for both groups are shown in figure 2 . The 5 -year RFS was $68 \%$ in the adjuvant chemotherapy group versus $77 \%$ in the group without adjuvant treatment (log-rank test, $\mathrm{p}=$ 0.638; HR: 1.2; 95\% CI, 0.5-2.8).

A total of 22 patients died during the follow-up period. There was no statistically significant difference in OS between the adjuvant chemotherapy group and the group without adjuvant treatment, with 5-year survival rates of 70 and $78 \%$, respectively (log-rank test, $\mathrm{p}=0.714 ; \mathrm{HR}: 1.2$; 95\% CI, 0.5-2.8).

In subgroup analysis, optimally and nonoptimally staged patients were analyzed separately. There was no association between improved survival and adjuvant chemotherapy after optimal staging (RFS: HR, 1.2; 95\% CI, 0.2-10.1, and OS: HR, 3.6; 95\% CI, 0.9-14.0) or adjuvant chemotherapy after nonoptimal staging (RFS: HR, 0.6; 95\% CI, 0.2-1.7, and OS: HR, 0.7; 95\% CI, 0.2-2.4). How-

Table 3. Characteristics of optimally staged patients compared to nonoptimally staged patients

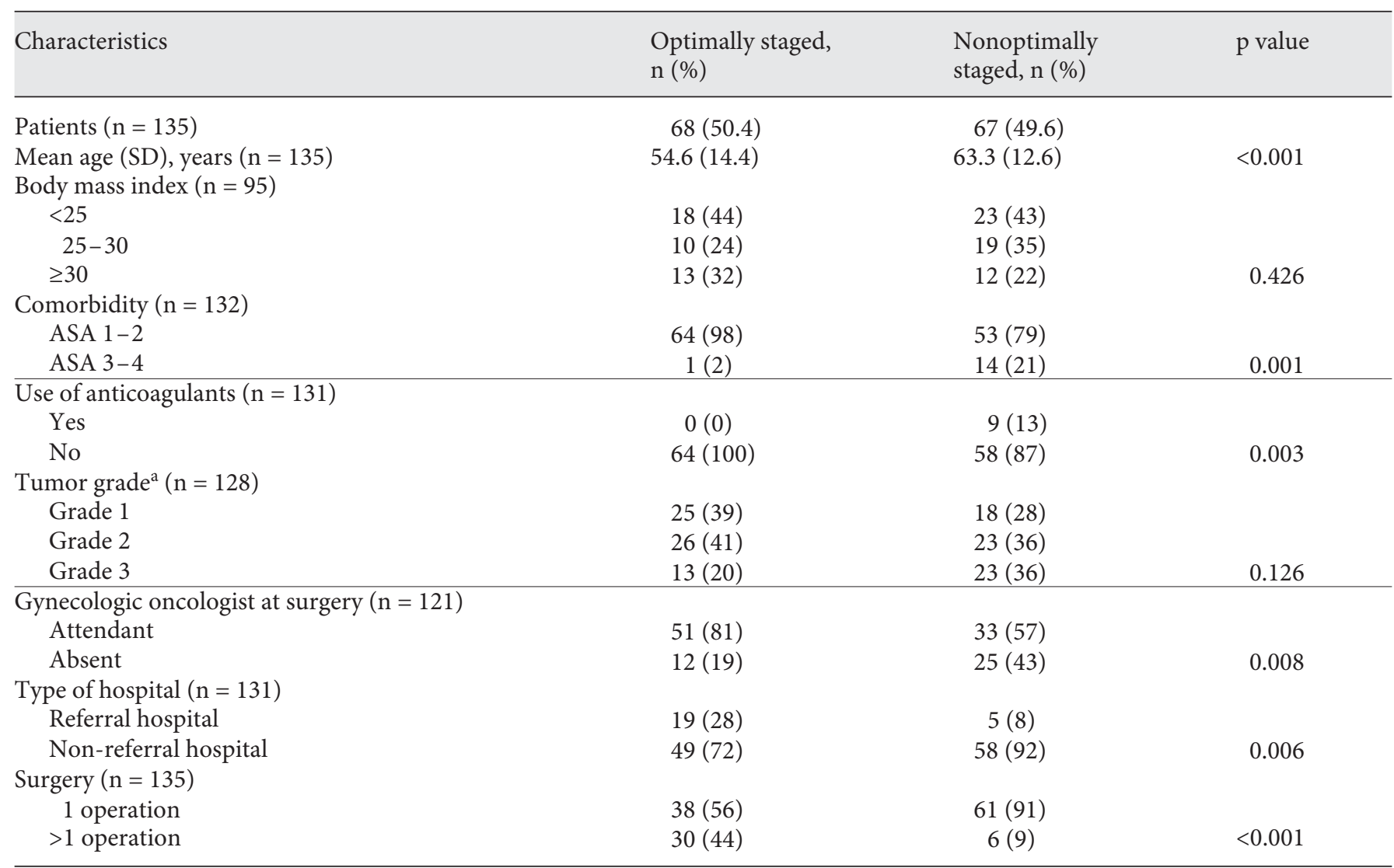

ASA = American Society of Anesthesiology; ASA 1-2 = healthy or mild systemic disease; ASA 3-4: moderate-severe systemic disease. Anticoagulants used were vitamin K antagonists, heparin or low- molecular-weight heparin. ${ }^{\text {a }}$ World Health Organization grading system. 


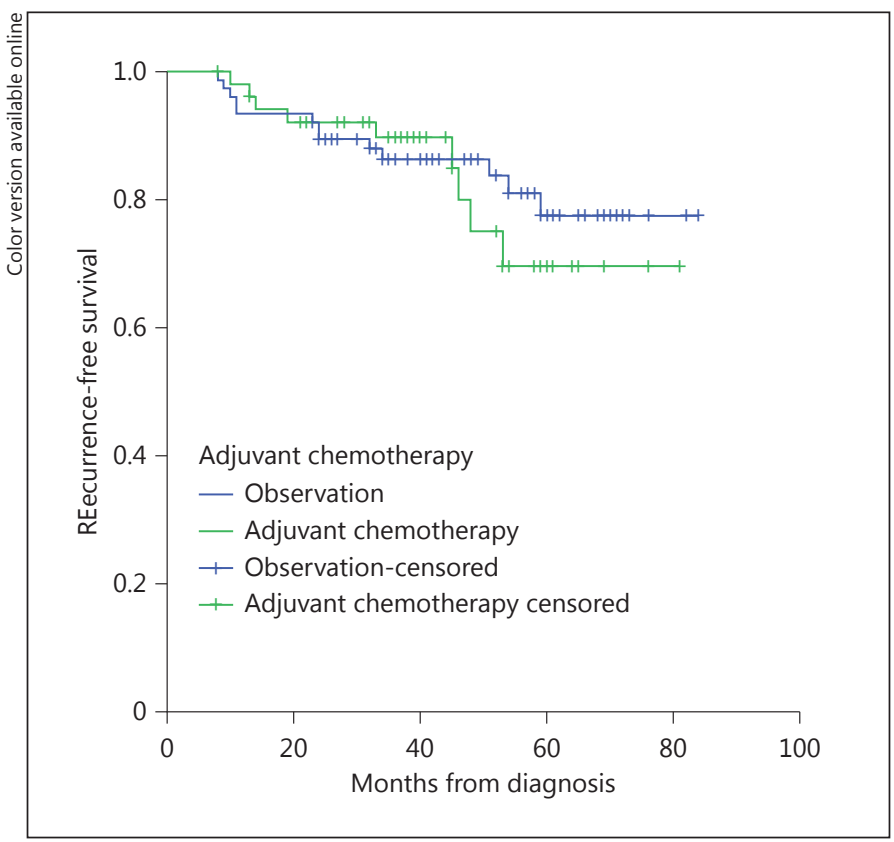

Fig. 2. Kaplan-Meier curves of RFS in all early-stage EOC patients. Adjuvant chemotherapy compared to no adjuvant treatment.

ever, patients with grade 3 tumors showed a significant positive association with improved RFS in the adjuvant chemotherapy group, with a 5-year survival rate of $90 \%$ in the adjuvant chemotherapy group compared to $46 \%$ in the group without adjuvant treatment $(\log$-rank test, $\mathrm{p}=$ 0.041 ). This positive association in the adjuvant chemotherapy group was maintained after Cox regression analysis with correction for the staging procedure (HR, 5.7; $95 \%$ CI, 1.0-30.8). The 5-year OS of patients with grade 3 tumors was $94 \%$ in the adjuvant chemotherapy group compared to $44 \%$ in the group without adjuvant treatment (log-rank test, $\mathrm{p}=0.005)$. After correction for staging, the adjuvant chemotherapy group showed improved OS compared to the group without adjuvant treatment (HR, 12.1; 95\% CI, 1.3-115).

\section{Discussion}

The aim of the present study was to determine clinical practice of the application of adjuvant chemotherapy in patients with early-stage EOC. In this cohort of 135 patients, $40 \%$ received adjuvant chemotherapy. Adjuvant chemotherapy was more frequently applied in patients with higher FIGO stage, clear-cell histology and a nonop- timal staging procedure. There was no difference in outcome between patients with and without adjuvant chemotherapy, except for patients with grade 3 tumors.

Despite the fact that several publications address the use of adjuvant chemotherapy in early-stage EOC, studies analyzing results obtained in clinical practice are lacking. A previous Dutch study by Vernooij et al. [10] described the use of adjuvant chemotherapy in $71-85 \%$ of patients with indications for adjuvant chemotherapy and in 64$77 \%$ of all ovarian cancer patients. However, both earlyand advanced-stage ovarian cancer patients were included in this analysis. In 2006, Sijmons et al. [11] published a retrospective cohort study analyzing adherence to guidelines in early-stage EOC patients in the Netherlands. In their study, it was shown that $10 \%$ of optimally staged patients and $7 \%$ of nonoptimally staged patients received chemotherapy. Yet, only patients with grade 2 and 3 tumors were included, since grade 1 tumors were not considered for any adjuvant therapy. Interestingly, no differences were observed in survival between patients with or without adjuvant treatment regardless of the staging procedure. More recently, Dinkelspiel et al. [12] investigated the use and duration of adjuvant chemotherapy in early-stage ovarian cancer patients in correlation with survival outcomes among low- and high-risk patients. Among low-risk patients (i.e. stage Ia or Ib, grade 1 or 2 and no clear-cell histology), 32.9\% received adjuvant chemotherapy versus $71.9 \%$ of high-risk patients (i.e. stage Ic, grade 3 or clear-cell histology). The use of adjuvant chemotherapy was associated with high tumor grade and stage, and was less likely to be applied in patients with advanced age and high comorbidity. In the recently updated Cochrane review, the authors concluded that adjuvant platinum-based chemotherapy results in an improved survival in women with early-stage EOC. To date, it remains uncertain whether women with low- and intermediate-risk early-stage disease will benefit as much from adjuvant chemotherapy as women with high-risk disease [8].

One of the most important factors determining the indication for adjuvant chemotherapy is the completeness of surgical staging $[6,7]$. Sixty-eight patients $(50 \%)$ in our study underwent an optimal staging procedure, which is in line with previously published studies that report optimal staging in $24-60 \%$ of patients with early-stage ovarian cancer, while some report much poorer percentages of optimal staging $[7,10,13,14]$.

With respect to the criteria for the staging procedure, there are differences in the literature. In the study by Vernooij et al. [10], patients who underwent at least one peri- 
toneal biopsy and had at least one acquired lymph node were considered as optimally staged, whereas the ICON1 trial recommended hysterectomy, bilateral salpingo-oophorectomy and omentectomy as the minimum procedure $[6,10]$. Several previous trials investigated the number and regions of lymph node sampling [15-20]. These trials showed that sampling of $\geq 10$ lymph nodes is necessary to detect occult metastasis and bilateral disease, and to improve the 5-year OS. By contrast, another study reported that adjuvant treatment after minimal surgery (i.e. without routine nodal sampling and peritoneal biopsy) has no effect on survival rates [21]. In our study, prognostic factors for nonoptimal staging were advanced age, high comorbidity and treatment in nonreferral hospitals. Whether these risk factors are reasons for omitting an adequate staging procedure remained unclear, since the majority of these patients received adjuvant chemotherapy. Treatment in referral hospitals and/or the attendance of a gynecologic oncologist at the time of surgery were associated with a high rate of optimal staging. These findings are in accordance with other studies [10, 13, 2224].

Recurrent disease was detected in 19\% of the patients, which is consistent with the recurrence rates reported in other studies (10-30\%) [6, 7, 13, 25-27]. In the present study, adjuvant chemotherapy was not significantly associated with RFS and OS regardless of the staging procedure. The ACTION and ICON1 trials showed significantly improved survival rates in patients receiving adjuvant chemotherapy. Specifically, the ACTION trial showed improved survival after adjuvant chemotherapy in nonoptimally staged patients. However, in the present study, the baseline characteristics of patients who did, or did not receive, adjuvant chemotherapy differed significantly with respect to FIGO stage, tumor grade and histology, whereas in the ACTION and ICON1 trials, baseline characteristics were similar in both arms. Patients who did not receive adjuvant chemotherapy in our cohort had more favorable tumor characteristics, i.e. stage 1a, grade 1 and clear-cell histology, than patients who received adjuvant chemotherapy. These characteristics were not comparable to the baseline characteristics of the patients in the ACTION and ICON1 trials, which explains why patients in the observation arm in the ACTION and ICON1 trials had a worse prognosis than patients who did not receive adjuvant chemotherapy in our cohort. Theoretically, this could lead to more advantageous effects of adjuvant chemotherapy in the ACTION and ICON1 trials, which cannot be demonstrated in our study, because of its retrospective nature. Sijmons et al. [11] and Dinkelspiel et al.
[12] reported no significant benefits of adjuvant chemotherapy with respect to survival rates, which is in accordance with our data $[11,12]$.

Although our study did not detect an association between adjuvant treatment and improved survival in the overall population, in patients with grade 3 tumors, adjuvant chemotherapy was associated with improved longterm effects. The 10-year results of the ICON1 study, which were published recently, showed an advantageous long-term effect of adjuvant chemotherapy in high-risk tumors [27].

The present study had several limitations, and one of the most important ones was its retrospective design. Differences between the outcomes of patients who did and did not receive adjuvant chemotherapy could be associated with the baseline characteristics rather than the chemotherapy treatment. Moreover, the study setting did not consider the upstaged patients. In addition, the accuracy of all staging procedures in GOCS could not be fully assessed. Another limitation of the study was the small effective sample size of the cohort and, in particular, the number of patients with grade 3 tumors.

Nonetheless, the present study provides an overview of the clinical practice in the treatment of early-stage EOC in the Netherlands and demonstrates reasons for deviating from standard care. Although randomized controlled trials provide the most accurate evidence of the effectiveness of a particular treatment, it is still important to consider the clinical setting and applicability. Ideally, every patient with early-stage EOC should be optimally staged; however, in routine clinical practice, a substantial proportion of patients undergo suboptimal surgical treatment. Furthermore, patients with indications for adjuvant chemotherapy are, in practice, often omitted from adjuvant treatment. Lee et al. [14] recently investigated the adherence to guidelines with respect to patient outcomes in early-stage EOC in Korean patients and showed guideline adherence in $26.7 \%$ of 266 patients. Treatment in accordance with guidelines led to significantly improved survival outcomes. The fact that $84 \%$ of the patients in our study were treated according to Dutch guidelines indicates that the GOCS provides high-quality care for patients with early-stage EOC in the Netherlands, but long-term complications of chemotherapy-induced neuropathy should also be considered and balanced especially for patients with low- and intermediate risk $[8,9,28]$.

In conclusion, $40 \%$ of the patients with early-stage EOC received adjuvant chemotherapy. The use of adjuvant chemotherapy was associated with high tumor stage, 
clear-cell histology and nonoptimal staging. Selective treatment of high-risk patients might explain the comparable outcome of patients with and without chemotherapy and supports a more individualized treatment approach in early-stage EOC.

\section{Acknowledgments}

We thank R.H. Hermans, Catharina Hospital, Eindhoven, M.C. Vos, St. Elisabeth Hospital, Tilburg, H.W.H. Feyen (deceased), Amphia Hospital, Breda, A.F.G.M. van de Walle, Elkerliek Hospital, Helmond, C.M. Engelhart, St. Anna Hospital, Geldrop, S.A. ter Haar, Maxima Medical Center, Veldhoven, and J.J. van Beek, Vie Curi Medical Center Venlo, for their contribution to the data collection.

\section{References}

1 IKNL: Incidentie Eierstokkanker. 2011, http://cijfersoverkanker.nl/selecties/Dataset_2/img50aa07960dc4c.

2 Sant M, Chirlaque Lopez MD, Agresti R, Sánchez Pérez MJ, Holleczek B, Bielska-Lasota M, et al: EUROCARE-5 Working Group. Survival of women with cancers of breast and genital organs in Europe 1999-2007: results of the EUROCARE-5 study. Eur J Cancer 2015, pii: S0959-8049(15)00702-9.

3 Prat J; FIGO Committee on Gynecologic Oncology: Staging classification for cancer of the ovary, fallopian tube, and peritoneum. Int J Gynaecol Obstet 2014;124:1-5.

4 Simard EP, Ward EM, Siegel R, Jemal A: Cancers with increasing incidence trends in the United States: 1999 through 2008. CA Cancer J Clin 2012;62:118-128.

5 IKNL: Overleving Eierstokkanker. 2011, http://cijfersoverkanker.nl/selecties/dataset_1/img50ad00ba86897.

6 Colombo N, Guthrie D, Chiari S, Parmar M, Qian W, Swart AM, et al; International Collaborative Ovarian Neoplasm (ICON) Collaborators: International Collaborative Ovarian Neoplasm trial 1: a randomized trial of adjuvant chemotherapy in women with earlystage ovarian cancer. J Natl Cancer Inst 2003; 95:125-132.

7 Trimbos JB, Vergote I, Bolis G, Vermorken JB, Mangioni C, Madronal C, et al; EORTCACTION Collaborators. European Organisation for Research and Treatment of CancerAdjuvant ChemoTherapy in Ovarian Neoplasm: Impact of adjuvant chemotherapy and surgical staging in early-stage ovarian carcinoma: European Organisation for Research and Treatment of Cancer-Adjuvant ChemoTherapy in Ovarian Neoplasm trial. J Natl Cancer Inst 2003;95:113-125.

8 Lawrie TA, Winter-Roach BA, Heus P, Kitchener HC: Adjuvant (post-surgery) chemotherapy for early stage epithelial ovarian cancer. Cochrane Database Syst Rev 2015; 12:CD004706.

9 Ezendam NP, Pijlman B, Bhugwandass C, Pruijt JF, Mols F, Vos MC, et al: Chemotherapy-induced peripheral neuropathy and its impact on health-related quality of life among ovarian cancer survivors: results from the population-based PROFILES registry. Gynecol Oncol 2014;135:510-517.
10 Vernooij F, Heintz AP, Coebergh JW, Massuger LF, Witteveen PO, van der Graaf Y: Specialized and high-volume care leads to better outcomes of ovarian cancer treatment in the Netherlands. Gynecol Oncol 2009;112:455461.

11 Sijmons EA, van Lankveld MA, Witteveen PO, Peeters PH, Koot VC, van Leeuwen JS: Compliance to clinical guidelines for earlystage epithelial ovarian cancer in relation to patient outcome. Eur J Obstet Gynecol Reprod Biol 2007;131:203-208.

12 Dinkelspiel HE, Tergas AI, Zimmerman LA, Burke WM, Hou JY, Chen L, et al: Use and duration of chemotherapy and its impact on survival in early-stage ovarian cancer. Gynecol Oncol 2015;137:203-209.

13 Timmers PJ, Zwinderman AH, Coens C, Vergote I, Trimbos JB: Understanding the problem of inadequately staging early ovarian cancer. Eur J Cancer 2010;46:880-884.

14 Lee JY, Kim TH, Suh DH, Kim JW, Kim HS, Chung $\mathrm{HH}$, et al: Impact of guideline adherence on patient outcomes in early-stage epithelial ovarian cancer. Eur J Surg Oncol 2015; 41:585-591.

15 Skírnisdóttir I, Sorbe B: Lymph node sampling is of prognostic value in early stage epithelial ovarian carcinoma. Eur J Gynaecol Oncol 2005;26:181-185

16 Carnino F, Fuda G, Ciccone G, Iskra L, Guercio E, Dadone D, et al: Significance of lymph node sampling in epithelial carcinoma of the ovary. Gynecol Oncol 1997;65:467-472.

17 Onda T, Yoshikawa H, Yokota H, Yasugi T, Taketani Y: Assessment of metastases to aortic and pelvic lymph nodes in epithelial ovarian carcinoma. A proposal for essential sites for lymph node biopsy. Cancer 1996;78:803808.

18 Negishi H, Takeda M, Fujimoto T, Todo Y, Ebina $\mathrm{Y}$, Watari $\mathrm{H}$, et al: Lymphatic mapping and sentinel node identification as related to the primary sites of lymph node metastasis in early stage ovarian cancer. Gynecol Oncol 2004;94:161-166.
19 Cass I, Li AJ, Runowicz CD, Fields AL, Goldberg GL, Leuchter RS, Lagasse LD, Karlan BY: Pattern of lymph node metastases in clinically unilateral stage I invasive epithelial ovarian carcinomas. Gynecol Oncol 2001;80:56-61.

20 Suzuki M, Ohwada M, Yamada T, Kohno T, Sekiguchi I, Sato I. Lymph node metastasis in stage I epithelial ovarian cancer. Gynecol Oncol 2000;79:305-308.

21 Beena A, Howells R, Lutchman-Singh K, Dhar K, Lim K, Jones R, et al: Simplified staging laparotomy in FIGO stage 1 epithelial ovarian cancer: follow-up and outcomes in south Wales, U.K. J Obstet Gynaecol 2013;33: 888-891.

22 Mayer AR, Chambers SK, Graves E, Holm C, Tseng PC, Nelson BE, et al: Ovarian cancer staging: does it require a gynecologic oncologist? Gynecol Oncol 1992;47:223-227.

23 van Altena AM, van den Akker PA, de Hullu JA, Ottevanger PB, Aalders AL, et al: Efficacy of a regional network for ovarian cancer care. Obstet Gynecol 2013;122:668-675.

24 Giede KC, Kieser K, Dodge J, Rosen B: Who should operate on patients with ovarian cancer? An evidence-based review. Gynecol Oncol 2005;99:447-461.

25 Gadducci A, Cosio S, Zola P, Sostegni B, Fuso L, Sartori E: Prognostic factors and clinical outcome of patients with recurrent earlystage epithelial ovarian cancer: an Italian multicenter retrospective study. Int J Gynecol Cancer 2013;23:461-468.

26 Colombo N, Van Gorp T, Parma G, Amant F, Gatta G, Sessa C, et al: Ovarian cancer. Crit Rev Oncol Hematol 2006;60:159-179.

27 Collinson F, Qian W, Fossati R, Lissoni A, Williams C, Parmar M, et al: ICON1 collaborators. Optimal treatment of early-stage ovarian cancer. Ann Oncol 2014;25:1165-1171.

28 Ezendam NP, Pijlman B, Bhugwandass C, Pruijt JF, Mols F, Vos MC, Pijnenborg JM, van de Poll-Franse LV: Chemotherapy-induced peripheral neuropathy and its impact on health-related quality of life among ovarian cancer survivors: results from the population-based PROFILES registry. Gynecol Oncol 2014;135:510-517. 